\title{
High k Dielectric Elastomeric Materials for Low Voltage Applications
}

\author{
C. Walder ${ }^{a}$, M. Molberg ${ }^{\mathrm{a}, \mathrm{b}}$, D. M. Opris ${ }^{\mathrm{a}}$, F. A. Nüesch ${ }^{\mathrm{a}}$, C. Löwe ${ }^{\mathrm{a} *}$, C. J.G. Plummer ${ }^{\mathrm{b}}$, \\ Y. Leterrier ${ }^{\text {b }, ~ J .-A . ~ E . ~ M a ̊ n s o n ~}{ }^{\text {b }}$ \\ a Laboratoy for Functional Polymers, Swiss Federal Laboratories for Material Testing \\ and Research (Empa), CH-8600 Dübendorf, ZH, Switzerland. \\ b Laboratoire de Technologie des Composites et Polymères (LTC), École \\ Polytechnique Fédérale de Lausanne (EPFL), CH-1015 Lausanne, VD, Switzerland
}

\begin{abstract}
In principle EAP technology could potentially replace common motion-generating mechanisms in positioning, valve control, pump and sensor applications, where designers are seeking quieter, power efficient devices to replace conventional electrical motors and drive trains. Their use as artificial muscles is of special interest due to their similar properties in terms of stress and strain, energy and power densities or efficiency. A broad application of dielectric elastomer actuators (DEA) is limited by the high voltage necessary to drive such devices.

The development of novel elastomers offering better intrinsic electromechanical properties is one way to solve the problem. We prepared composites from cross-linked silicone elastomers or thermoplastic elastomers (TPE) by blending them with organic fillers exhibiting a high dielectric constant. Well characterized monomeric phthalocyanines and modified doped polyaniline (PANI) were used as filler materials. In addition, blends of TPE and an inorganic filler material PZT were characterized as well. We studied the influence of the filler materials onto the mechanical and electromechanical properties of the resulting mixtures. A hundredfold increase of the dielectric constant was already observed for blends of an olefin based thermoplastic elastomer and PANI.
\end{abstract}

Keywords: dielectric elastomer, electroactive polymer, mechanical properties, polymer blend

\section{INTRODUCTION}

Dielectric elastomer actuators are electric field driven polymer transducers [1,2], which have exceptional properties such as large strains (up to $380 \%$ ), fast response ( $>1 \mathrm{KHz}$ ), lightweight, noise free actuation and inexpensive processing. The soft materials can be used to design novel sensors, actuators and generators for applications ranging from robotics, prosthetics to optics. A variety of demonstrators based on DEA have been realized [3], however only a few products are currently on the market $[4,5]$. DEA are based on the electromechanical response of an elastomer film (typically silicone or acrylic polymers) with compliant electrodes on each surface. When a voltage is applied an electrostatic pressure is built up, the almost incompressible film shrinks in thickness and expands in area; electrical energy is directly converted into mechanical energy.

The simple working principle of actuators based on dielectric elastomer technology is shown in figure 1. Using a simple electrostatic model (opposite charges on the electrodes attract each other, while the like charges on the electrodes repel each other so called Maxwell stress), we can derive the effective pressure produced by the electrodes on the film as a function of the applied voltage. This pressure, $p$, is

$\mathrm{p}=\varepsilon_{\mathrm{r}} \varepsilon_{0} \mathrm{E}^{2}=\varepsilon_{\mathrm{r}} \varepsilon_{0}(\mathrm{~V} / \mathrm{t})^{2} \quad$ equation 1

$\varepsilon_{\mathrm{r}}$ and $\varepsilon_{0}$ are the permittivity of free space and the relative permittivity (dielectric constant) of the polymer, respectively. $\mathrm{E}$ is the applied electric field; $\mathrm{V}$ is the applied voltage; and $\mathrm{t}$ is the film thickness.

*christiane.loewe@empa.ch; phone 414482344 99; fax 4144 8; empa.ch

Electroactive Polymer Actuators and Devices (EAPAD) 2009, edited by Yoseph Bar-Cohen, Thomas Wallmersperger, Proc. of SPIE Vol. 7287, 72870Q - (C) 2009 SPIE · CCC code: 0277-786X/09/\$18 - doi: 10.1117/12.815926 

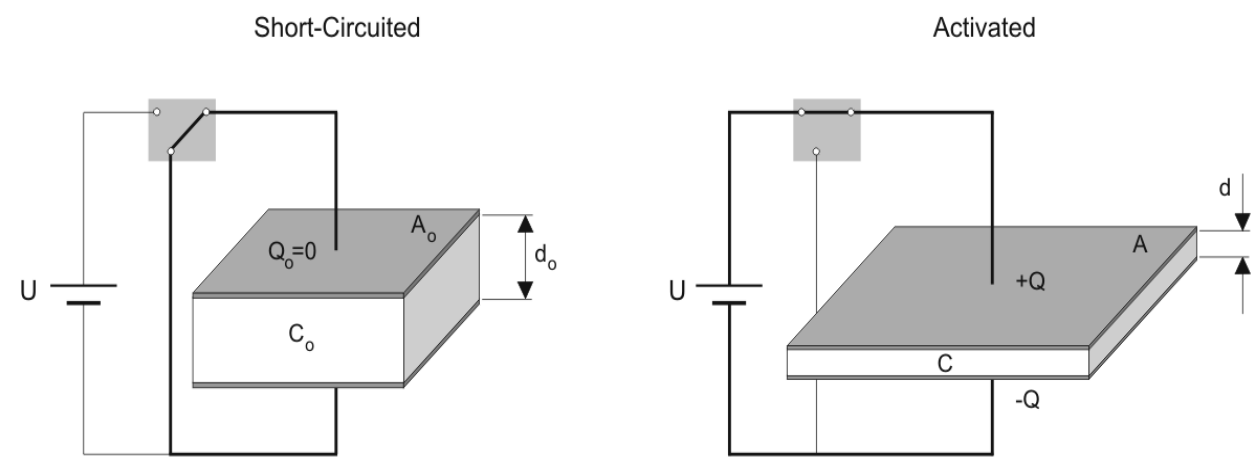

Figure 1: Functional element of dielectric elastomer actuators. Polymer film compresses in thickness and expands in area when a voltage is applied across the film.

Main obstacles for most applications are the high actuation voltages ( $\mathrm{kV}$ range) and the limited long term behavior of DEAs influenced by their stress relaxation, hysteresis, creep and viscoelastic properties. Especially, the need of lower actuation voltage requires tailor made elastomers with good overall electro-mechanical properties [6]. Enhancing the material's dielectric constant $\varepsilon$ and reducing the thickness of the elastomeric film are the most promising approaches to lower the required drive voltage by one to two decades to values between 40 and $600 \mathrm{~V}$. Therefore, we want to develop optimized novel elastomers and blend them with organic fillers exhibiting high dielectric constants [7].

\section{RESULTS AND DISCUSSION}

Research for better dielectric materials with higher dielectric constants and material strength is pursued. The latter determines the elastic ability of the material to withstand the electrostatic forces which are appearing. A high dielectric constant generates larger electrostatic forces accounting for actuation stress and strain. Blending of high dielectric fillers, such as phthalocyanines, conductive metals or polymers, into the elastomer matrix was proven a promising approach to enhance $\varepsilon$ of the resulting composites $[8,9]$. However, adding fillers and additives to the polymer matrix may affect negatively other properties of the elastomer such as mechanical properties or electric breakdown field. The overall performance may even be diminished instead of enhancing the electro mechanical response. Elastomer material NBR (acrylonitrile butadiene rubber) has emerged as a promising material comprising a reasonable high dielectric constant of 14 and a significantly higher strength (about $3 \mathrm{MPa}$ ) when compared to an acrylic based elastomer (VHB 4910 from 3M) [10], although the stress and strain levels observed for the VHB could not jet be reached by NBR. We investigated a soft thermoplastic elastomer gel [11] for its potential as dielectric elastomer in electromechanical transducers. Choosing a thermoplastic material adds up the desirable thermoplastic processing and offers reshaping of films or devices. Cross linked elastomers likely VHB or PDMS are lacking such advantages.

\subsection{Dielectric elastomer materials}

A capable dielectric material for DEA technology is characterized by several main properties:

- low elastic modulus $(<1 \mathrm{MPa})$

- good mechanical properties such as elongation at break (a few $100 \%$ ), tensile strength at break (>4 MPa)

- high entropy elasticity with a low viscoelastic part

- High permittivity (up to 100 )

- High electric breakdown field $(>80 \mathrm{~V} / \mu \mathrm{m})$

- Good chemical resistance and aging behaviour 
In a first step we compared mechanical properties of the common polyacrylate (VHB 4910 from 3M), mostly used in DEA technology, with cross linked polydimethylsiloxane (PDMS) and a thermoplastic olefin based elastomer gel (TPE). The tensile tests were performed with a Zwick (Z010) machine. For the thermoplastic TPE a similar run of the stress/strain curve, a smooth increase of stress upon stretching up to $600 \%$ is observed, when compared to the polyacrylate elastomer, while the PDMS built up stress (refer figure 2). While rupture of the cross linked PDMS occurs about $400 \%$ strain, the thermoplastic material ruptures at about $2100 \%$ and can be elongated almost 3 times more than the widely used acrylic VHB 4910 material, which breaks at strains of about $700 \%$. The stress/strain curve for a sample of TPE filled with a 5\% weight fraction of a doped polyaniline (TPE $+5 \%$ Pani), is little influenced compared to the non filled sample.

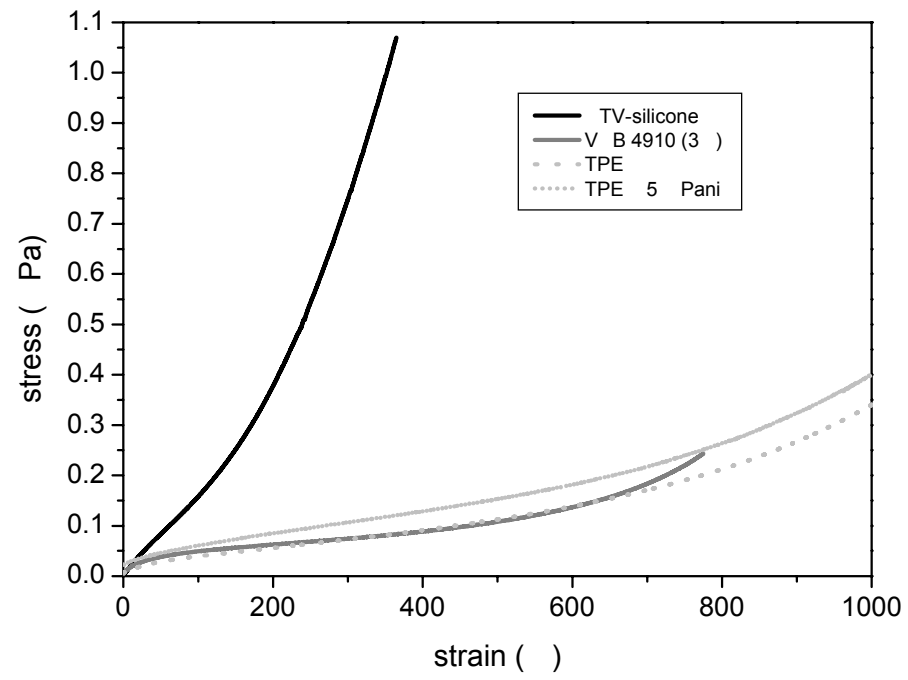

Figure 2: Tensile curves of a cross linked PDMS and thermoplastic TPE compared with acrylic VHB 4910. The tensile curve of a polymer blend from TPE with Polyaniline $5 \%(\mathrm{w} / \mathrm{w})$ is also depicted.

TPE shows a lower modulus than VHB concomitant with a significant higher tensile strength and strain. If the true cross section is taken into account an even higher (15fold,) tensile strength is observed for TPE (refer to table 1). While VHB behaves like a typical rubbery elastic material, at a higher strain the thermoplastic material is not breaking as a typical cross linked elastomer but cracks occur and the strength diminish under plastic deformation before final rupture. Lower modulus and higher strength and strain make the TPE material in principle more attractive as dielectric elastomer than the polyacrylate VHB 4910 considering static one dimensional mechanical properties.

Table 1: Modulus at $100 \%$, strength, stress and stain at break for TPE and VHB 4910.

\begin{tabular}{|l|l|l|l|l|}
\hline Material & $\begin{array}{l}\text { Modulus } \\
\text { at 100\% } \\
{[\mathrm{MPa}]}\end{array}$ & $\begin{array}{l}\text { Tensile strength at } \\
\text { break } \\
\text { nominal true }\end{array}$ & $\begin{array}{l}\text { Stress at break } \\
{[\mathrm{MPa}]}\end{array}$ & $\begin{array}{l}\text { Strain at break } \\
{[\%]}\end{array}$ \\
\hline VHB 4910 & 0.07 & $0.34 \quad$ ca. 4.6 & 0.34 & 680 \\
\hline TPE & 0.05 & $2.34 \quad$ ca. 77 & 1.93 & 2060 \\
\hline
\end{tabular}

In circular actuators or other actuator designs the planar expansion of the dielectric elastomeric film is of interest. Therefore we built a simple setup to measure the biaxial deformation of clamped dielectric elastomer films under pneumatic pressure. In figure 3 the lower stiffness of the linear strain upon biaxial deformation for samples based on TPE is also demonstrated compared to cross linked PDMS as well as an acrylic based material. 


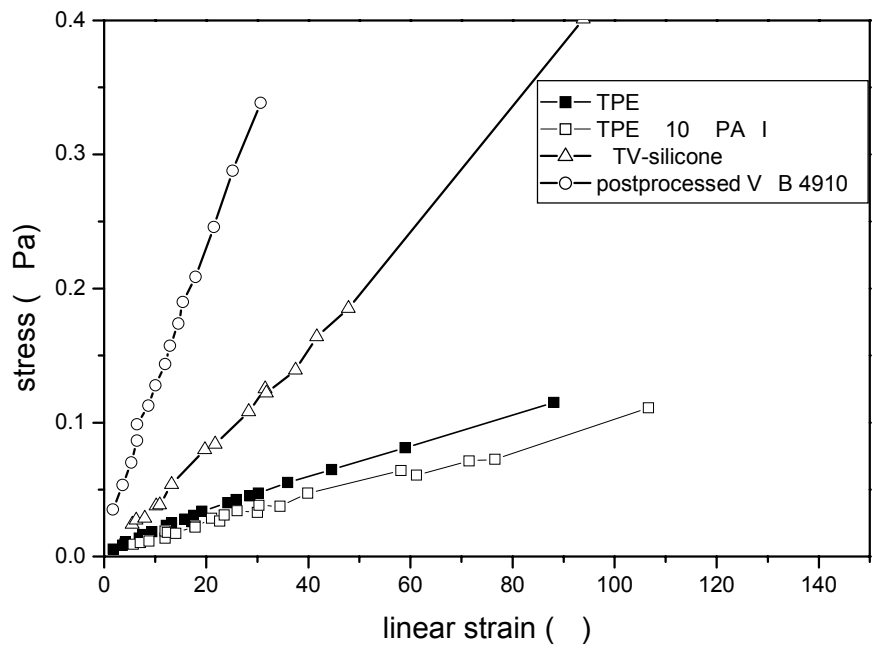

Figure 3: linear strain of 2 D deformation cross linked PDMS and TPE compared to VHB 4910 (modified). The curve for a polymer blend sample of TPE with Polyaniline $(10 \%(\mathrm{w} / \mathrm{w}))$ is shown as well.

In addition to the static short term mechanical properties, stress relaxation experiments were performed. Under a constant strain of one dimensional tension the relaxation of the original strain was observed in function of time. The geometry of the samples for VHB 4910 and TPE were adapted in such a way that an original strain of $50 \%$ was obtained. Measurement were undertaken on a dynamic mechanical spectrometer Type ARES and lasted for $100 \mathrm{~h}$. From the resulting data a trend line was extrapolated up to $1000 \mathrm{~h}$. Relaxation phenomena can lead to buckling or distortion of pre-stressed actuators and reduce their shelf lifetime drastically, or influence their electromechanical performance, which make it complicated to control such devices. Typically, pre-stressed films are applied to diminish their thickness in order to achieve same strain and stress levels at lower electric fields, enhance the electric breakdown field by reorienting chemical bonds and defects, reduce response speed or induce preferential expansion when asymmetrically stretched or lessen boundary effects from passive materials. As negative consequences pre-straining reduces actuator power density, increase risk of stress concentration and failure as well as stiffening of the material, which leads to reduced strain response. Ease of fabrication is limited and supporting structures are necessary to keep the material in a pre-strained state.

VHB

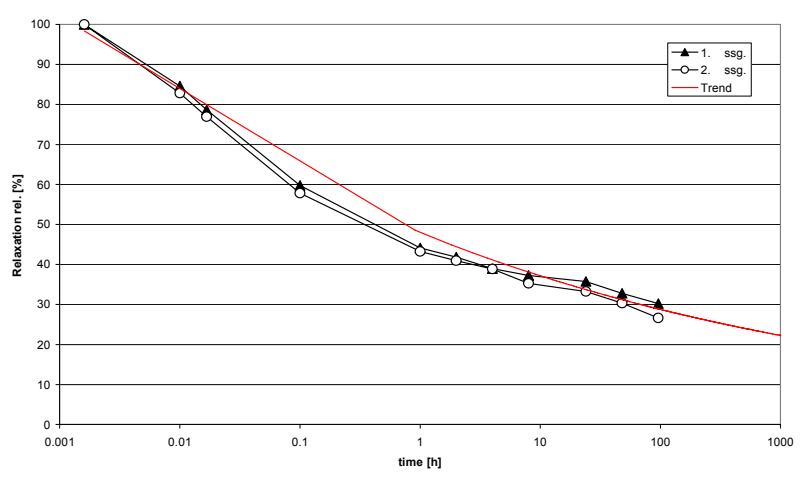

TPE-Gel

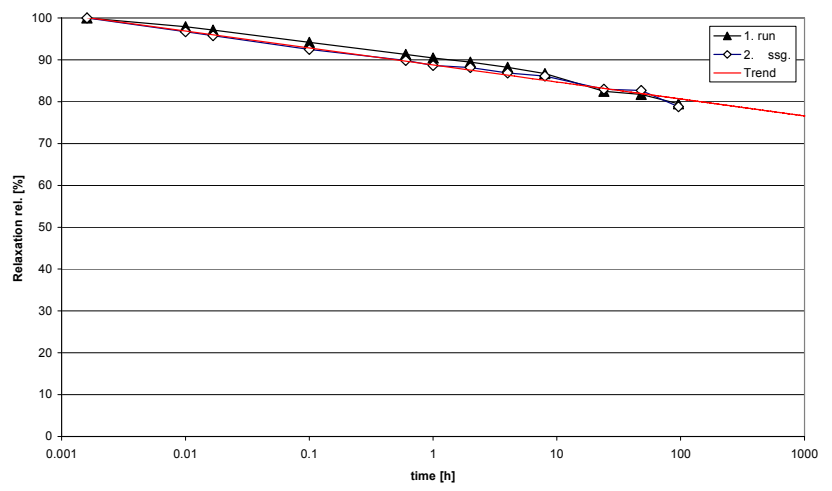

Figure 4: Relative relaxation in function of time for VHB 4910 (left) and TPE (right) 
The results of the relaxation experiments are summarized in figure 4. Examine the relaxation behavior after $1000 \mathrm{~h}$ (extrapolated) the modulus is still about $75 \%$ of the original modulus for TPE, while in the same time span the modulus for VHB 4910 is strongly affected and reduced to about $25 \%$ of the original value. Again the chosen TPE seems to exhibit superior properties than VHB 4910 although a reduction of $25 \%$ of mechanical properties upon relaxation phenomena is still quite high.

Torsion pendulum tests on TPE and VHB 4910 were performed to determine the dynamic shear and loss modulus as well as damping behavior in dependence of time. Characteristic data such as glass transition and flow temperature of amorphous polymers are obtained and the range of the short term service temperature can be described.

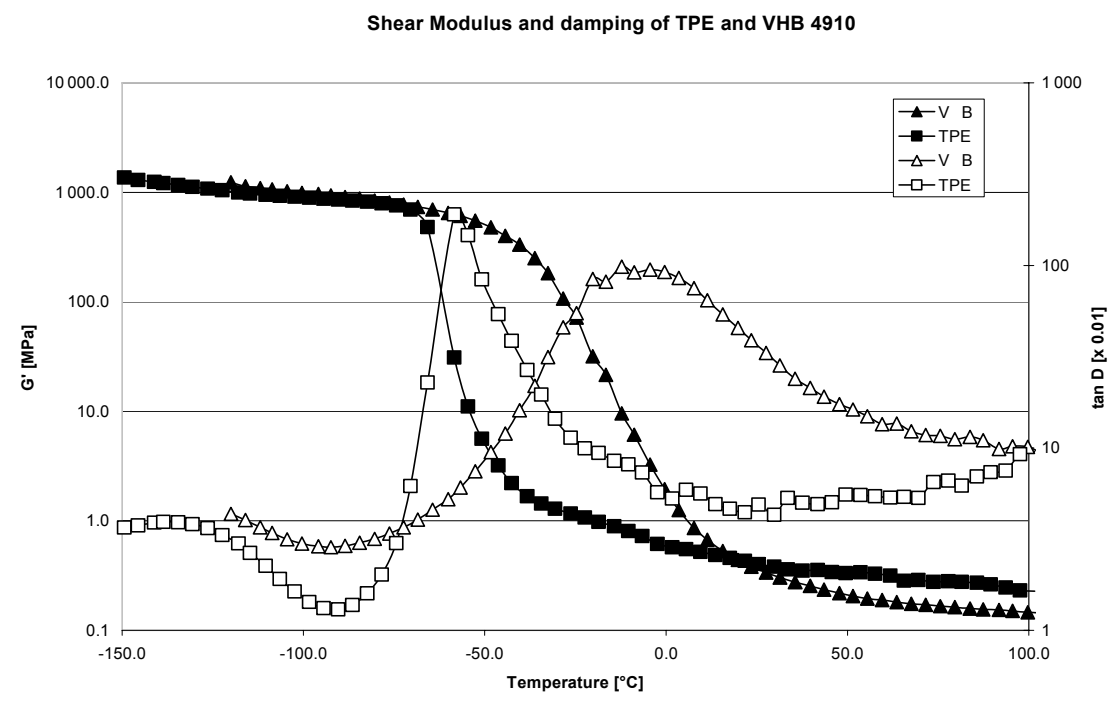

Figure 5: Shear modulus (full symbol) and $\tan \delta$ (empty symbol) of VHB 4910 (triangle symbol) and TPE (square symbol), respectively.

VHB 4910 shows a broad glass transition ranging from $-60^{\circ} \mathrm{C}$ to $+50^{\circ} \mathrm{C}$. The maximum of the damping can be observed at $-4.4^{\circ} \mathrm{C}$. The regime of the service temperature can be estimated between $+10^{\circ} \mathrm{C}$ and $80^{\circ} \mathrm{C}$ with an accompanying relatively high loss factor of 0.6 to 0.1 .

With $-57^{\circ} \mathrm{C}$ (see figure 5, maximum of tan $\delta$ ) the glass transition point of TPE is much lower and the features of the peak are rather narrow compared to the shear modulus and $\tan \delta$ curves of VHB 4910. Therefore, the regime for the service temperature is estimated to be between -25 and $+80^{\circ} \mathrm{C}$ concomitant with a much lower loss factor of 0.1 to 0.06 .

Shear modulus and damping behavior of VHB 4910 is characteristic for an adhesive with good vibration damping properties, given the fact, that VHB 4910 was developed for such applications, this finding is not astonishing. For use in DEAs with dynamic exposure the high and strongly temperature dependent loss factor within the rather narrow service temperature regime is not suitable and excludes application of VHB 4910 based actuators at low temperatures. With an about $50 \mathrm{~K}$ lower glass transition temperature and a lower modulus within the service temperature regime accompanied with a 10 fold lower loss factor, again the thermoplastic TPE exhibits better dynamic mechanical features and more appropriate temperature behavior for applications in a DEA than VHB 4910.

In summary, the chosen thermoplastic elastomer exhibits with a low modulus concomitant with a relatively high strength, especially, when the true strength is taken into account, and an extraordinary maximum strain good $1 \mathrm{D}$ and $2 \mathrm{D}$ mechanic properties, which meet the required criteria. In addition relaxation phenomena are relatively low compared to VHB 4910 but there is still room for improvement. With a glass transition at ca. $-57^{\circ} \mathrm{C}$ and a lower loss factor applications at lower temperatures are getting more feasible with the proposed TPE material than the common used VHB 4910. 
Finally, electromechanical response of circular actuators was determined for unfilled TPE and cross linked PDMS in comparison to VHB 4910. Although the electric breakdown field of TPE is lower than for silicones or VHB4910, same strain levels can be reached for TPE at lower electric fields and therefore at lower actuation voltage (compare to figure $6)$.

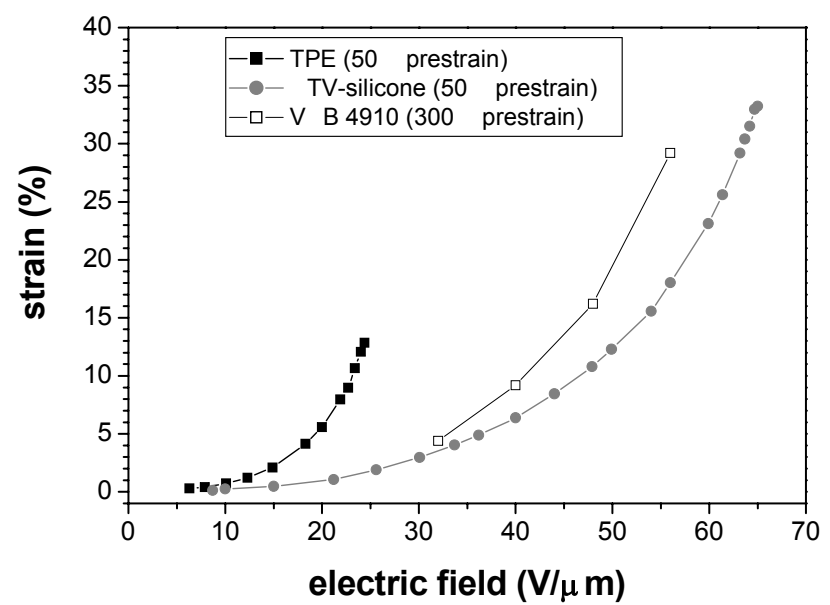

Figure 6: Electromechanical response of circular actuators of TPE, PDMS and VHB 4910

\subsection{Polymer blends with high $\mathrm{k}$ filler materials}

We investigated blends from cross-linked PDMS and thermoplastic TPE as polymer matrix and well defined monomer metal phthalocyanines (Pc), doped polyaniline (PANI) or polyvinylbenzene encapsulated PANI and PZT ceramics (lead zirconium titanate) as filler materials. While cross-linking of PDMS is hindered by PANI or Pc fillers and no good films could be prepared, mixtures of PANI or Pc with TPE lead to significantly higher $\varepsilon$ for the blended material when compared to unfilled TPE. We started with polyaniline (PANI), which was doped with dodecyl benzene sulfonic acid (DBSA) in a molecular ratio of 1: 0.5 to receive an electrical conductive and in unpolar solvents soluble filler material. Due to the solubility of the filling material we could incorporate weight fractions up to $20 \%$ of the PANI/DBSA filler into the thermoplastic TPE matrix. With low fractions of 5 to $10 \%$ of the doped PANI in the blend, the dielectric constant of the silicone matrix, which is roughly 2 at low frequency is only moderately influenced (compare to figure 7). With a content of ca. $20 \%$ PANI in TPE the dielectric constant of the blend is raised to ca. 400 . This is about at 200 fold increase of the dielectric constant for the blended material compared to the unfilled material.

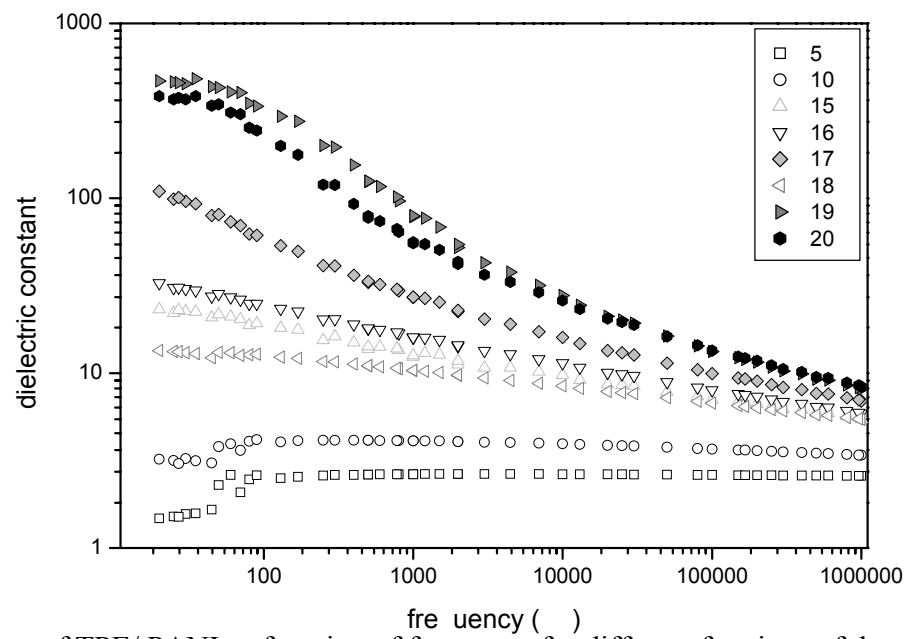

Figure 7: Dielectric constant of TPE/ PANI as function of frequency for different fractions of doped PANI. 
Due to increasing dielectric loss and decreasing breakdown field caused by agglomerated conductive particles the application of such blends as dielectric elastomer in actuators is limited. Moreover, we could recently demonstrate that the high $\varepsilon$ values for Pcs [12] originate from proton conductivity and water content rather than from specific structural and electronic characteristics of the Pcs [13]. Further investigations of encapsulated and modified (substitution of water by imidazole) phthalocyanines will demonstrate their potential as high $\mathrm{k}$ fillers.

Encapsulated PANI [14] and PZT ceramics were incorporated as particles via extrusion into the thermoplastic TPE matrix $[11,15]$. Volume fractions of 13,23 and $33 \%$ of the filler material could be mixed into TPE. The resulting concentrations in weigh for the mixtures are between 14 and $36 \mathrm{w} / \mathrm{w} \%$ for PANI and considerably higher for mixtures of PZT and TPE (56 to $81 \mathrm{w} / \mathrm{w} \%)$.

Influence of filler content onto mechanical properties is summarized in table 2 . To comprise the changes in stiffness the modulus at 100 and $200 \%$ strain is compared. In addition strain and strength at break from one dimensional tension tests are illustrating filler induced changes.

For blends of PZT and TPE a surprisingly little influence onto the modulus is observed. For the highest filling degree the modulus was increased only about 10 to $15 \%$. In as similar way strain is also little affected and diminished of about 16 $\%$ for the highest filler degree. On the other hand tensile strength is reduced with increasing filling degree. Mixtures of encapsulated PANI /TPE are already affected by low fractions of filler content and stiffen. With a fraction of $33 \mathrm{~V} \%$ of encapsulated PANI particles in TPE the modulus is increased of about 3.5 fold, while strain and strength are reduced to about $50 \%$ of the value of the unfilled material.

Table 2: Mechanical parameters and electric breakdown field of filled polymer blends of TPE and PZT and encapsulated PANI particles

\begin{tabular}{|l|l|l|l|l|l|l|l|}
\hline Material & TPE & \multicolumn{3}{l|}{ PZT/TPE blend } & \multicolumn{3}{l|}{ Enpasulated doped PANI/ TPE blend } \\
\hline $\begin{array}{l}\text { Filling degree } \\
\text { volume \% }\end{array}$ & 0 & 13 & 23 & 33 & 13 & 23 & 33 \\
\hline $\begin{array}{l}\text { Modulus@ } \\
100 \%[\mathrm{MPa}]\end{array}$ & 0.05 & 0.05 & 0.06 & 0.06 & 0.06 & 0.10 & 0.13 \\
\hline $\begin{array}{l}\text { Modulus@ } \\
200 \%[\mathrm{MPa}]\end{array}$ & 0.07 & 0.07 & 0.07 & 0.08 & 0.10 & 0.17 & 0.25 \\
\hline $\begin{array}{l}\text { Strength@ } \\
\text { break [MPa] }\end{array}$ & 2.34 & 2.4 & 1.9 & 1.6 & 1.7 & 1.5 & 1.2 \\
\hline $\begin{array}{l}\text { Strain@ } \\
\text { break [MPa] }\end{array}$ & 2060 & 1990 & 1780 & 1730 & 1780 & 1630 & 1100 \\
\hline $\begin{array}{l}\text { Electrical } \\
\text { breakdown } \\
{[\text { V/ } \mu \mathrm{m}]}\end{array}$ & $>50$ & ca. 30 & ca. 30 & ca. 30 & 16.5 & 18 & 8 \\
\hline
\end{tabular}

Permittivity of blends was determined as function of frequency with a LCR meter. In blends with the highest filler concentration a moderate enhancement of a factor of 5 for PZT filled materials and a factor of 7 for encapsulated PANI particles in the low frequency regime (about $20 \mathrm{~Hz}$ ) is observed, when compared to the unfilled TPE ( $\varepsilon$ about 2 ). For the PZT filler probably higher filling degrees are necessary to reach the percolation limit and hence a higher permittivity. Encapsulating of doped PANI results in better properties concerning electric loss, on the other hand augmentation of permittivity is quite low if compared to blends doped with not encapsulated PANI.

A further important characteristic for dielectric elastomer actuators is the electric breakdown field. We prepared films of 0.3 to $0.5 \mathrm{~mm}$ thickness and clamped them between frames, contacted the surfaces of the films with punctual electrodes, and connected the devices to a high voltage source. A rising electric field was applied until an electric breakthrough occurred or an electric current of $2 \mu \mathrm{m}$ was observed. The resulting electrical breakdown field are summarized in table 2 . 
Blending TPE with PTZ ceramics reduces the breakdown field down to $30 \mathrm{~V} / \mu \mathrm{m}$ for all mixtures. With enhancing filling degree the breakdown field is strongly reduced in case of the TPE/PANI blends.

\section{CONCLUSIONS}

A thermoplastic elastomer gel (a two phase system, block-copolymer with a high fraction of aliphatic oil), TPE was investigated as dielectric elastomer in electromechanical transducers. Intrinsic good mechanical properties (e.g. elastic modulus, tensile strain and strength), dynamic properties as shear modulus and low loss factor in addition with good relaxation behaviour and low glass transition temperature accompanied by a relatively broad service temperature range enables the novel material as appropriate dielectric material in DEA technology. Preparation of polymer blends of TPE and encapsulated PANI or PZT ceramic particles via thermoplastic processing is feasible. Surprisingly little influence of mechanical properties and electric breakdown field for TPE/PZT blends was observed. For mixtures of TPE/ encapsulated PANI elasticity, strain and strength were affected more drastically and are probably related to interactions of the free acid from not sufficiently encapsulated doped PANI with TPE.

\section{AKNOWLEGEMENT}

The study was undertaken within the Empa project "Artificial Sphincter Muscle" No. 841'331 and is funded by Empa. The authors thank B. Fischer for tensile tests. Dr H. Kramer is thanked for fruitful discussions.

\section{REFERENCES}

[1] Bar-Cohen, Y., World-Wide EAP: EAP Materials and Product Manufacturers, (2006) http://ndeaa.jpl.nasa.gov/nasa nde/lommas/eap/EAP-material-n-products.htm

[2] Carpi, F., DeRossi, D., Kornbluh R., Pelrine, R., Sommer-Larsen, P.(ed.) "Dielectric Elastomers as Electromechanical Transducers, Fundamentals, Materials, Devices, Models and Applications of an Emerging Electroactive Polymer Technology", Elsevier Ltd, (2008).

[3] Bar-Cohen, Y., (ed.) Electroactive polymer (EAP) actuators as artificial muscle, 2nd edition, SPIE Press, Bellingham, WA, (2004).

[4] www.optotune.com

[5] www.artificialmuscle.com

[6] Pelrine. R, Kornbluh, R., Pei, Q., Joseph, J., "High-speed electrically actuated elastomers with strain greater than $100 \%$," Science, 287, 836-839 (2000).

[7] Zhang, X., Löwe, C. Wissler, M., Jähne, B., Kovacs, G., "Dielectric Elastomers in Actuator Technology Adv. Eng. Mater. 5, 361-367 (2005).

[8] Huang, C., Zhang, Q. M., Li, J. Y., and Rabeony, M,"Colossal dielectric and electromechanic response in selfassembled polymeric nanocomposites," Appl. Phys. Lett., 87, 182901 (2005).

[9] Carpi, F., Gallone, G., Galantini, F., DeRossi, D., "Silicone-Poly(hexyl-thiopene) blends as elastomers with enhanced electromechanical transduction properties" Adv. Funct. Mat., 18 (2), 235-241 (2008).

[10] Jung, K., Lee, J.H., Cho, M.S., Koo, J., Nam, J., Lee, Y.K. et al., "Development of enhanced synthetic rubber for energy efficient polymer actuators", Smart structures and materials 2006: electroactive polymer actuators and devices (EAPAD). In: Bar Cohen, Y., ed. Proceedings of the SPIE, 6168, 157-165 (2006).

[11] Walder Ch., Entwicklung eines thermoplastischen Dielektrikums für elektromechanische Aktoren” Master Thesis, FH Nordwestschweiz Hochschule Technik, 2008.

[12] Nalwa, H.S., "Characterization and studies of electroactive properties of an organometallic polymer of ironphthalocyanine," Appl. Organomet. Chem., 2, 257-262 (1988).

[13] Opris, D.M., Nüesch, F., Löwe, Ch., Molberg, M., Nagel, M., "Synthesis, Characterization, and Dielectric Properties of Phthalocyanines with Ester and Carboxylic Acid Functionalities", Chem. Mater., 20 (21), 6889- 2896 (2008)

${ }^{[14]}$ D. Opris (private communication) 
[15] Opris, D.M., Molberg, M., Löwe, Ch., Nüesch, F., Plummer, C. J. G., Leterrier, Y., Månson, J.-A. E. Proc. of 9th biennial ASME Conference on Engineering Systems Design and Analysis ESDA, Haifa (Israel) (2008). 